\title{
Digital Watermarking using Discrete Wavelet Transform
}

\author{
Malika Narang \\ Department of electrical, \\ electronics \& communication \\ engineering, ITM University \\ Sec-23a, Gurgaon
}

\author{
Sharda Vashisth \\ Department of electrical, \\ electronics \& communication \\ engineering, ITM University \\ Sec-23a, Gurgaon
}

\begin{abstract}
Now days due to advancement of technology it is difficult to protect creative content and intellectual property. It is very easy to copy and modify digital media resulting in great loss in business. So the viable solution for this problem is digital watermarking. Digital watermarking is a technique by which we embed copyright mark into digital content which is used to identify the original creator and owner of digital media. It is prominently used for tracing copyright infringements. In this paper technique based on 1-level discrete wavelet transform is used for insertion and extraction of watermark in original image by using alpha blending. This technique is much simpler and robust than others.
\end{abstract}

\section{General Terms}

Digital watermarking, DWT

\section{Keywords}

Watermark embedding, Watermark extraction, Alpha blending

\section{INTRODUCTION}

Digital media can be copied and modified easily so protecting the copyright of digital media has become an important task. The digital watermark is introduced to solve the problem of copyright. The digital watermarking is a technique of embedding any watermark image into cover image using some known algorithm depending upon the requirement in multimedia data to identify the owner of the document[1].There are two common methods for watermarking: spatial domain and transform domain. In spatial domain pixels of an image are modified depending upon perceptual analysis of an image. But in transform domain some frequencies are selected and modified from their original values according to certain rules. The transform domain methods are more popular because watermark embedding is more robust in this domain as compared to spatial domain. It also provides more security and imperceptibility [3-5].In this paper we propose the watermarking scheme based on DWT (discrete wavelet transform) which works in transform domain. Watermarking algorithms are divided into two groups based on extraction: Blind and Non-blind watermarking. In blind watermarking extraction does not need original image but in non-blind watermarking original image is needed in watermark extraction [5].We use non-blind watermarking in this paper.

The paper is organized as follows. Section 2 contains watermarking scheme DWT .Section 3 and 4 contain proposed algorithms and section 5 contains experimental results.

\section{DISCRETE WAVELET TRANSFORM}

Discrete wavelet transform is a multiresolution decomposition of a signal. It hierarchically decomposes an image [2]. Mapping an image into a set of coefficients is done by discrete wavelet transform. Basically non stationary signals are processed by discrete wavelet transform. Both frequency and spatial domain of an image are provided by discrete wavelet transform i.e. it captures both frequency and location information. In this, decoding is done in low resolution to high resolution manner. High and low frequency parts are obtained on decomposing an image by dwt. Information about edge components are contained in high frequency parts and, low frequency parts again decomposed into another set of low and high frequency parts [2-3].The image is divided into four multiresolution subbands LL, LH, HL and HH using DWT. Fine-scale DWT coefficients are represented by $\mathrm{LH}, \mathrm{HL}, \mathrm{HH}$ sub-bands and coarse-scale DWT coefficients are represented by LL subbands. LL sub-band is further decomposed into four multiresolution sub-bands to obtain next coarser wavelet coefficients. This process is repeated several times determined by application for which it is used [10].

In this paper we used 1-level DWT which decomposes an image into LL1 which is lower resolution approximation coefficient and LH1, HL1, HH1 which are detailed components of an image [3].

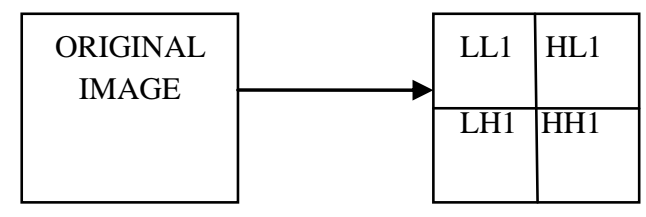

Figure 1: 1-level discrete wavelet decomposition

Discrete wavelet transform is used in many applications related to signal processing such as compression of audio and video, also used in noise removal. It provides high compression ratio with good quality of reconstruction.

\section{WATERMARK EMBEDDING}

To hide personal data into cover image in perceptually visible manner is the main purpose of proposed paper. A mathematical tool discrete wavelet transform is selected to achieve this object. The wavelet of daubecheis is used for watermarking. Wavelet domain watermarking is used because it reduces the risk of any distortions such as 
compression and low pass filtering that changes the high frequency components of an image but it cannot resist attacks that destroy the whole watermarked image such as cropping [5]. In this first we take cover image and decomposed it into four components i.e. low frequency approximation, high frequency diagonal, low frequency horizontal, low frequency vertical components using 2D DWT. The same procedure is applied on the watermark image which is to be embedded into cover image. Now alpha blending technique is used for inserting a watermark in cover image. In this first we apply dwt to both cover and watermark image to obtain decomposed components which are further multiplied by a particular scaling factor and are added [1] [9]. We cannot embed the watermark directly into cover image wavelet coefficients; we embed wavelet coefficients of watermark image into cover image because approximation wavelet coefficients contain more information of original watermark image [2]. Here we want watermark to be perceptible or visible in nature, so we embed watermark in low frequency approximation component of cover image [1] [3][8].

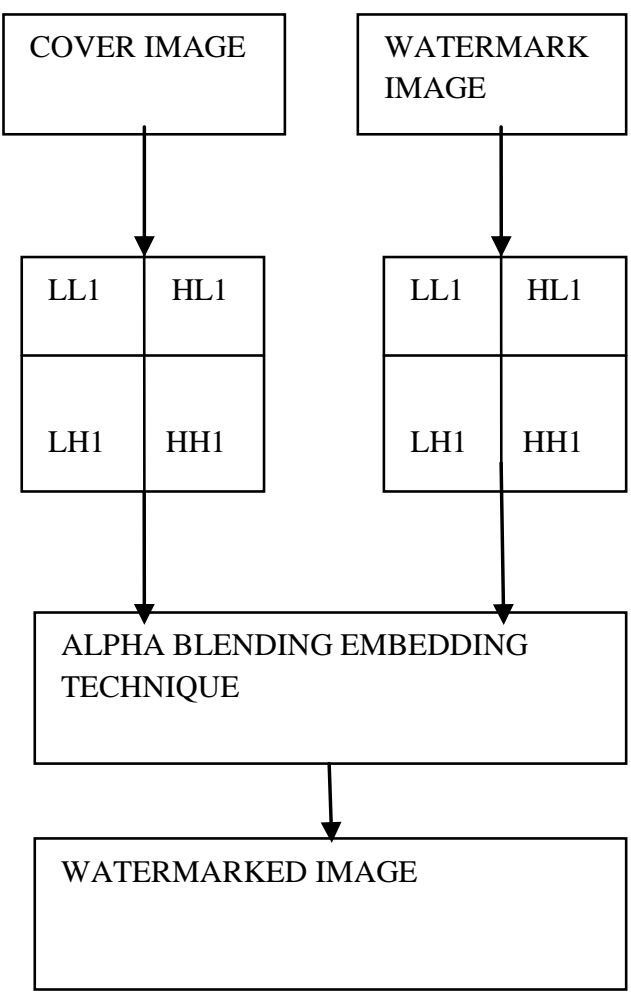

Figure 2: Watermark embedding technique

\subsection{Alpha blending}

Alpha blending is the process of combining an image with a background to create the appearance of partial or full transparency. The formula of alpha blending used for watermarking embedding is:

Watermarked image $=\mathrm{A}^{*}(\mathrm{LL} 1)+\mathrm{B} *(\mathrm{WM} 1)$

$\mathrm{A}, \mathrm{B}=$ scaling factors for cover and watermark image respectively.

LL1=low frequency approximation of cover image

$\mathrm{WM} 1=$ watermark image

Then to generate secure watermarked image inverse discrete transform is applied to watermarked image coefficient [3].

\section{WATERMARK EXTRACTION}

In this watermarked image and cover image both are decomposed into their sub bands by applying DWT to both images. Now we use cover image to extract the watermark image using non-blind watermarking [5]. Then alpha blending formula is applied to recover the watermark image form watermarked image. Here low frequency approximation component of cover image is first multiplied by a particular scaling factor and then subtracted from watermarked image coefficient [1] [3].

\subsection{Alpha blending}

The alpha blending formula used for watermark extraction is:

Recovered watermark $=(\mathrm{WM}-\mathrm{A} * \mathrm{LL} 1)$

WM=Watermarked image

$\mathrm{A}=$ scaling factor for cover image

LL1 = Low frequency approximation component of cover image

To generate the final watermark extracted image, inverse discrete transform is applied to watermark image coefficient.

\section{WATERMARK ED IMAGE}

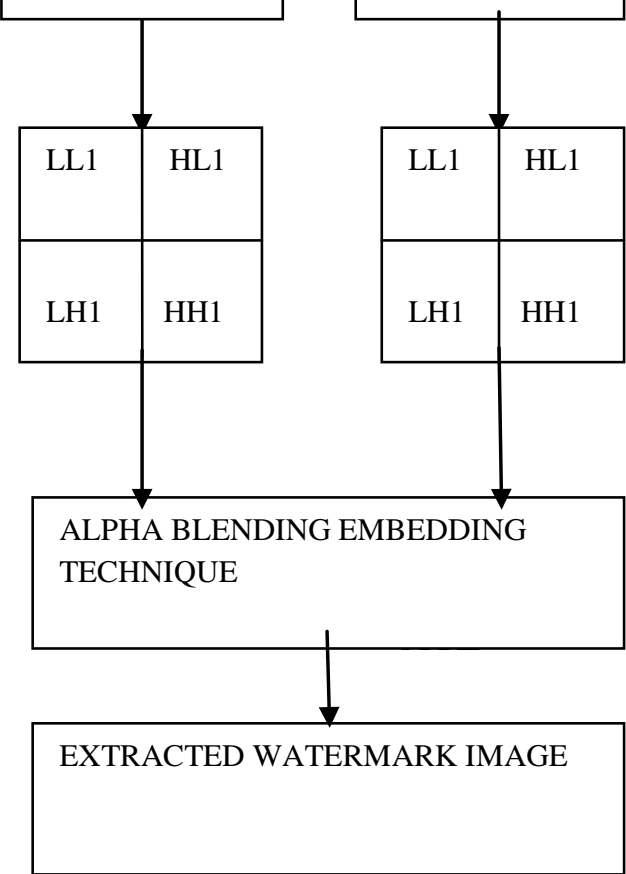

Figure 3: Watermark extraction technique 


\section{EXPERIMENTAL RESULTS}

Two grayscale images are used for performing watermarking process. One image is taken as cover image named boat image and other is taken as watermark image named fruit image. In this low frequency contents of both images are taken. This technique is called alpha blending. Both images are of equal size i.e. of $225 * 225$. The watermark image is embedded into cover image by varying the value of A from 1.0 to 0.4.But value of $\mathrm{B}$ remains constant. A gives the best results at 0.5. As the value of $\mathrm{A}$ decreases below 0.4 , watermark image becomes brighter and completely covers and destroys the cover image. For recovering the watermark image value of A varies from .9 to .4 depending upon the value of $\mathrm{A}$ in embedding algorithm. As value decreases below 0.2, recovered watermark become darker and watermark become completely invisible. In fig 6,7,8,9 watermarked images are shown depending upon various values of $\mathrm{A}$ and the recovered watermark shown in fig $10,11,12,13$ based on different values of scaling factor $\mathrm{A}$

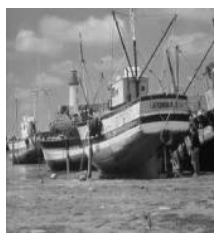

Figure 4: Boat (cover image)

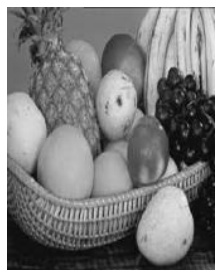

Figure 5: Fruit image (watermark)

\subsection{Watermarked images using various values of scaling factors $A$ and $B$}
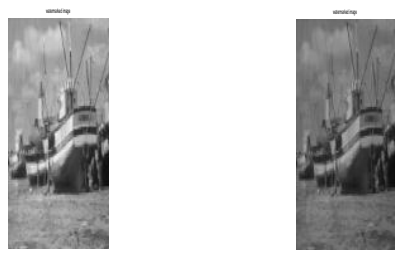

Figure: $6 \mathrm{~A}=1$

Figure: $7 \mathrm{~A}=.7$
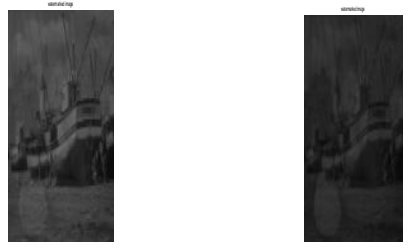

Figure: $8 \mathrm{~A}=.4$

Figure: $9 \mathrm{~A}=.2$

Note: scaling factor $\mathrm{B}=0.1$ for all watermarked images

\subsection{Recovered watermark for various values of scaling factor}
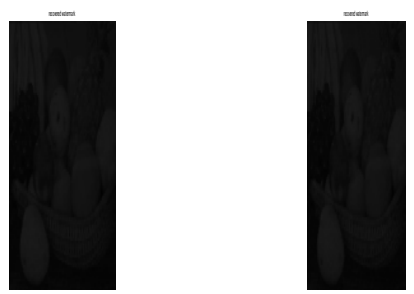

Figure: $10 \mathrm{~A}=.975$

Figure: $11 \mathrm{~A}=.675$
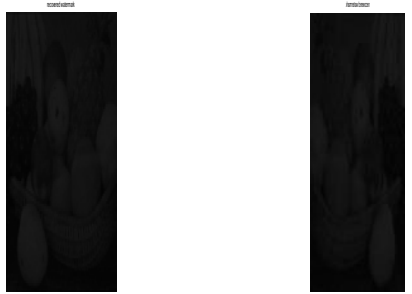

Figure: $12 \mathrm{~A}=.375$

Figure: $13 \mathrm{~A}=.175$ 
Table 1. Recovered watermark image for various values of $\mathrm{A}$ and $\mathrm{B}$

\begin{tabular}{|l|l|l|l|l|}
\hline S.NO & A & B & PSNR & observation \\
\hline 1 & .875 & .1 & $\begin{array}{l}+6.89249 \\
\mathrm{~dB}\end{array}$ & \\
\hline 2 & .775 & .1 & $\begin{array}{l}+6.89281 \\
\mathrm{~dB}\end{array}$ & \\
\hline 3 & .675 & .1 & $\begin{array}{l}+6.89421 \\
\mathrm{~dB}\end{array}$ & \\
\hline 4 & .575 & .1 & $\begin{array}{l}+6.89294 \\
\mathrm{~dB}\end{array}$ & \\
\hline 5 & .475 & .1 & $\begin{array}{l}+6.90955 \\
\mathrm{~dB}\end{array}$ & Best result \\
\hline 6 & .375 & .1 & $\begin{array}{l}+6.88968 \\
\mathrm{~dB}\end{array}$ & \\
\hline 7 & .275 & .1 & $\begin{array}{l}+6.89634 \\
\mathrm{~dB}\end{array}$ & \\
\hline 8 & .175 & .1 & $\begin{array}{l}+6.89267 \\
\mathrm{~dB}\end{array}$ & \\
\hline 9 & & .75 & .1 & $\begin{array}{l}+5.78253 \\
\end{array}$ \\
\hline
\end{tabular}

We can also embed the watermark using 2-level DWT.It gives better results than 1-level.Now we compare proposed 1-level DWT from 2-level DWT.In 2-level DWT we further
Table 2. Watermarked image for various values of $A$ and

\begin{tabular}{|l|l|l|l|}
\hline S.NO & A & B & observation \\
\hline 1 & 1.0 & .1 & \\
\hline 2 & .9 & .1 & \\
\hline 3 & .8 & .1 & \\
\hline 4 & .7 & .1 & \\
\hline 5 & .6 & .1 & \\
\hline 6 & .5 & .1 & Best result \\
\hline 7 & .4 & .1 & \\
\hline 8 & .3 & .1 & \\
\hline 9 & .2 & .1 & \\
\hline 10 & .1 & .1 & \\
\hline
\end{tabular}

decompose the low level approximation coefficient i.e. LL1 obtained from 1-level DWT into 4 sub bands i.e. LL2, LH2, HL2, HH2.Here also we embedd the watermark coefficient into LL2 sub band of cover image. It gives better PSNR value then 1-level DWT as we see in table given below.

Table 3. Comparison of 1-level DWT and 2-Level DWT for Recovered watermark image

\begin{tabular}{|l|l|l|l|l|l|}
\hline S.no & A & B & $\begin{array}{l}\text { PSNR(1- } \\
\text { level) }\end{array}$ & PSNR(2-level) & observation \\
\hline 1 & .875 & .1 & $+6.89249 \mathrm{db}$ & $6.89255 \mathrm{db}$ & \\
\hline 2 & .775 & .1 & $+6.89281 \mathrm{db}$ & $+6.89290 \mathrm{db}$ & \\
\hline 3 & .675 & .1 & $+6.89421 \mathrm{db}$ & $+6.89427 \mathrm{db}$ & \\
\hline 4 & .575 & .1 & $+6.89294 \mathrm{db}$ & $+6.89300 \mathrm{db}$ & \\
\hline 5 & .475 & .1 & $+6.90955 \mathrm{db}$ & $+6.91002 \mathrm{db}$ & Best result \\
\hline 6 & .375 & .1 & $+6.88968 \mathrm{db}$ & $+6.88975 \mathrm{db}$ & \\
\hline 7 & .275 & .1 & $+6.89634 \mathrm{db}$ & $+6.89640 \mathrm{db}$ & \\
\hline 8 & .175 & .1 & $+6.89267 \mathrm{db}$ & $+6.89275 \mathrm{db}$ & \\
\hline 9 & .75 & .1 & $+5.78253 \mathrm{db}$ & $+5.78259 \mathrm{db}$ & \\
\hline
\end{tabular}




\section{CONCLUSION}

In this paper watermarking is done by dwt which is multiresolution technique and the decoding done is hierarchal [6]. In this proposed method visible watermark is embed into the cover image and also extracted from cover image with the help of cover image. The insertion and extraction of watermark is achieved by DWT (discrete wavelet transform), IDWT (inverse discrete transform) and the alpha blending technique. The scaling factors A, B determines the quality of watermarked image and recovered watermark

\section{ACKNOWLEDGMENTS}

Authors express their sincere gratitude to the researchers those who developed the various algorithms used in this article.

\section{REFERENCES}

[1] Akhil Pratap Singh, Agya Mishra, "Wavelet Based Watermarking On Digital Image", Indian Journal of Computer Science and Engineering, Vol.1, No.2, 8691

[2] Satendra Kumar, Jaydeep Kishore, Nitin Arora,"Enhanced Digital Image Watermarking Scheme Based On DWT and SWD",International Journal of Computer Applications, Vol.57, No.11, 2012.

[3] Nikita Kashyap, Sinha G.R,'Image Watermarking Using 2-Level DWT", Advances in Computational Research, Vol.4, Issue 1, 2012.
[4] Kamran Hameed, Adeel Mumtaz, S.A.M.Gilani,"Digital Image Watermarking in the Wavelet Transform Domain", World Academy of Science, Engineering and Technology 13, 2008.

[5] Mohammad Reza Soheili,"A Robust Digital Image Watermarking Scheme Based on DWT, Journal of Computer Engineering 1, 2009.

[6] Xiang-Gen Xia, Charles G. Boncelet, Gonzalo R. Arce,"Wavelet Transform Based Watermark for Digital Images", Optical Express 497, Vol. 3, No.12, 1998.

[7] NagarajV.Dharwadkar,B.B.Amberker,"Watermarking Scheme for Color Images using Wavelet Transform based Texture Properties and Secret Sharing", International Journal of Information and communication

[8] Amol R. Madane, K.T Talele,M.M Shah, "Watermark Logo in Digital Image using DWT, Proceedings of SPIT-IEEE Colloquium and International Conference,Vol.1.

[9] Nikita Kashyap, G.R. Sinha," Image Watermarking Using 3-Level Discrete Wavelet Transform (DWT)", I.J.Modern Education and Computer Science, Vol.3, 2012.

[10] Nasseer Moyasser Basheer, Shaimaa Salah Abdulsalam," Digital Image Watermarking Algorithm in Discrete Wavelet Transform Domain Using HVS Characteristics", 